\title{
Adaptation of Hansenula polymorpha to methanol: a transcriptome analysis
}

Tim van Zutphen ${ }^{1}$, Richard JS Baerends ${ }^{1}$, Kim A Susanna', Anne de Jong ${ }^{2}$, Oscar P Kuipers², Marten Veenhuis ${ }^{1,3}$, Ida J van der Klei ${ }^{1,3^{*}}$

\begin{abstract}
Background: Methylotrophic yeast species (e.g. Hansenula polymorpha, Pichia pastoris) can grow on methanol as sole source of carbon and energy. These organisms are important cell factories for the production of recombinant proteins, but are also used in fundamental research as model organisms to study peroxisome biology. During exponential growth on glucose, cells of $H$. polymorpha typically contain a single, small peroxisome that is redundant for growth while on methanol multiple, enlarged peroxisomes are present. These organelles are crucial to support growth on methanol, as they contain key enzymes of methanol metabolism.

In this study, changes in the transcriptional profiles during adaptation of H. polymorpha cells from glucose- to methanol-containing media were investigated using DNA-microarray analyses.

Results: Two hours after the shift of cells from glucose to methanol nearly 20\% (1184 genes) of the approximately 6000 annotated $H$. polymorpha genes were significantly upregulated with at least a two-fold differential expression. Highest upregulation (> 300-fold) was observed for the genes encoding the transcription factor Mpp1 and formate dehydrogenase, an enzyme of the methanol dissimilation pathway. Upregulated genes also included genes encoding other enzymes of methanol metabolism as well as of peroxisomal $\beta$-oxidation.

A moderate increase in transcriptional levels (up to 4-fold) was observed for several PEX genes, which are involved in peroxisome biogenesis. Only PEX11 and PEX32 were higher upregulated. In addition, an increase was observed in expression of the several ATG genes, which encode proteins involved in autophagy and autophagy processes. The strongest upregulation was observed for ATG8 and ATG11.

Approximately 20\% (1246 genes) of the genes were downregulated. These included glycolytic genes as well as genes involved in transcription and translation.

Conclusion: Transcriptional profiling of $H$. polymorpha cells shifted from glucose to methanol showed the expected downregulation of glycolytic genes together with upregulation of the methanol utilisation pathway. This serves as a confirmation and validation of the array data obtained. Consistent with this, also various PEX genes were upregulated. The strong upregulation of ATG genes is possibly due to induction of autophagy processes related to remodeling of the cell architecture required to support growth on methanol. These processes may also be responsible for the enhanced peroxisomal $\beta$-oxidation, as autophagy leads to recycling of membrane lipids. The prominent downregulation of transcription and translation may be explained by the reduced growth rate on methanol ( $t_{d}$ glucose $1 \mathrm{~h}$ vs $\mathrm{t}_{d}$ methanol $4.5 \mathrm{~h}$ ).
\end{abstract}

\footnotetext{
* Correspondence: i.j.van.der.klei@rug.nl

${ }^{1}$ Molecular Cell Biology, University of Groningen, P.O. Box 14, 9750 AA Haren,

the Netherlands
} 


\section{Background}

Hansenula polymorpha is an important cell factory for the production of pharmaceutical proteins [1]. Moreover, it is extensively used in fundamental research aiming at understanding the molecular principles of peroxisome biology [2].

As cell factory, $H$. polymorpha has several important advantages. First, it contains very strong and inducible promoters derived from the methanol metabolism pathway. Also, the organism is thermotolerant (it can grow at high temperatures up to $48^{\circ} \mathrm{C}$, [3]) and tolerates various environmental stresses. $H$. polymorpha does not hyperglycosylate secreted proteins, which often is a problem in heterologous protein production in S. cerevisiae.

In $H$. polymorpha peroxisomes massively develop during growth on methanol as sole source of carbon and energy. Methanol is oxidized by the enzyme alcohol oxidase (AOX), which is localized in peroxisomes together with catalase and dihydroxyacetone synthase (DHAS), the first enzyme of the assimilation pathway. Peroxisomes are not required for primary metabolism when cells are grown on glucose. Moreover, glucose represses the key enzymes of methanol metabolism AOX and DHAS. Therefore, during growth on glucose $H$. polymorpha cells contain only a single, small peroxisome. Upon a shift to methanol media, the enzymes of methanol metabolism are induced paralleled by an increase in peroxisome size and abundance. The initial small peroxisome serves as the target organelle for the enzymes of methanol metabolism and proliferates by fission [4]. Ultimately, in exponentially growing cells, each cell contains several enlarged peroxisomes [5].

A wealth of information is now available of individual genes encoding enzymes of the methanol metabolism as well as on genes involved in peroxisome formation (PEX genes). However, genomics approaches in $H$. polymorpha are still rare.

We used genome-wide transcriptional profiling to dissect the initial events accompanying the adaptation of glucose grown $H$. polymorpha cells to methanol metabolism. This will gain information on the induction and repression of metabolic genes as well as on non-metabolic genes, including PEX genes.

\section{Results and discussion}

All experiments described in this paper were performed in batch cultures. We chose not to grow the cells in carbonlimited chemostats, as glucose-limitation results in derepression of genes involved in methanol metabolism [6].

$H$. polymorpha cells were extensively pre-cultivated in batch cultures on mineral media supplemented with glucose as sole carbon source in order to fully repress the enzymes of methanol metabolism. Glucose cultures in the late exponential growth phase were transferred to fresh mineral medium containing methanol as sole carbon and energy source. As shown in figure 1, RT-PCR indicated that the inoculum cells (from the glucose batch culture at the late exponential growth phase, $\mathrm{OD}_{660 \mathrm{~nm}} 2.3$ ) did not contain transcript of alcohol oxidase $(\mathrm{AOX})$ or dihydroxyacetone synthase (DHAS), key enzymes of methanol metabolism. However, two hours after the shift to medium containing methanol, mRNAs of both genes were readily detected, a time-point which has also been identified as threshold for the detection of first AOX enzyme activity [5]. Therefore, 2 hours incubation on methanol was selected as sampling point of cells for transcriptome analysis.

Replicates were obtained by growing 4 independent cultures on glucose that were independently transferred to fresh media containing methanol. Of each culture, mRNA isolated from the glucose and the methanol sample was labeled (and dye-swapped) and hybridized on two arrays per culture. In addition, as a control $A O X$ transcript levels of these samples were determined by RT-PCR, confirming the absence of transcript in the glucose-grown pre-cultures and the presence of $A O X$ transcript after 2 hours incubation (data not shown).

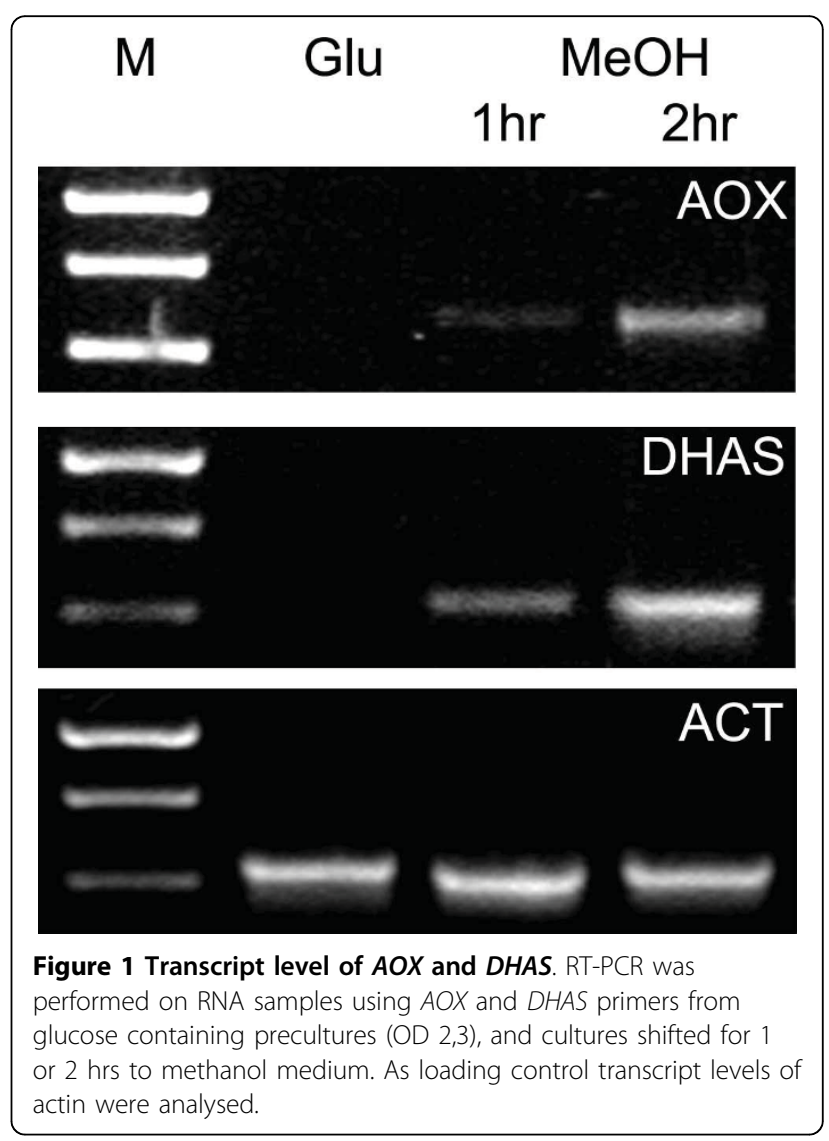




\section{Overview of the DNA microarray data}

The DNA microarray analyses data were analyzed to generate the ratio between the transcripts on methanol and glucose for each gene to identify any differential expression and to determine the p-value to assess the significance and the A-value to check the intensity of the signals. [Additional file 1: Supplemental table S1] presents an overview of the array results. Of the nearly 6000 annotated $H$. polymorpha genes that are listed, approximately $20 \%$ (1184 genes) are upregulated, while another 20\% (1246 genes) are downregulated with at least a two-fold differential expression, meeting the significance and signal intensity criteria.

Of the upregulated genes, 13 are more than 100 times upregulated, 192 genes show a 10-100-fold upregulation, 156 genes increase between 5 and 10-fold and the remaining 823 genes are less than 5 -fold upregulated. Highest upregulated are the central methanol metabolism regulator MPP1 (394-fold, Hp27g360, see below) and the gene encoding formate dehydrogenase (347fold), required in methanol catabolism. Also the other components of the methanol metabolic pathway are highly upregulated. Moreover, $C R C 1$ is highly induced, encoding a mitochondrial inner membrane carnitine transporter, which is required for the transport of acetyl-CoA from peroxisomes to mitochondria during fatty acid beta-oxidation (111-fold). In line with CRC1, also the genes involved in the beta-oxidation of fatty acids are overrepresented among the highly upregulated genes (for details see below). Furthermore, approximately $13 \%$ of the more than 10-fold upregulated genes is involved in transport. The upregulation of hexose transporters may be important for the uptake of the residual glucose that was present in the inoculum. Of the downregulated genes, the highest fold downregulation (65-fold) is observed for Hp24g956, encoding a protein with strong similarity to Sik1p of $S$. cerevisiae, which is involved in pre-rRNA processing. This predicted function is consistent with the general trend among the downregulated genes, since of the 179 genes that are over 10 -fold downregulated, nearly $50 \%$ code for products that function in either transcription or translation processes. Of the other downregulated genes, 269 show a 5- to 10 -fold downregulation. Of these, nearly $40 \%$ encode proteins involved in transcription and translation. The other 789 genes are less than 5 -fold reduced in transcript levels.

\section{Functional overview DNA microarray data - FUNCATS}

To obtain an overview of the functions of the differentially expressed genes, these were categorized according to the Functional Catalogue, FUNCAT $[7,8]$. In this system, each gene is classified in one or more groups, depending on its function. The number of genes in each category is shown as the percentage of the total number of up- or downregulated genes in the diagrams shown in figure 2. For comparison, a diagram showing the contribution of each functional category to the total number of genes in $H$. polymorpha is included. To construct this diagram, all known $H$. polymorpha genes are used; both up- and downregulated genes as well as non-regulated genes.

As expected, genes involved in metabolic pathways strongly contribute to both the up- and downregulated genes ( $20 \%$ and $15.5 \%$, respectively), reflecting the largescale adaptations accompanying the shift from glucose to methanol. However, metabolism is a large group also in the total genome and the contribution in percentages does not reflect the nature of the metabolic pathways that are regulated (see below).

In contrast to metabolism, some other functional categories display a difference in contribution to the upregulated compared to the downregulated genes. One such functional category is the group of protein synthesis genes, which is almost absent among upregulated genes $(0.25 \%)$, while it composes a large portion of the downregulated genes $(12.1 \%)$. Of the total genome of $H$. polymorpha only approximately $4 \%$ is involved in protein synthesis, reflecting the considerable effect of a shift to methanol on protein synthesis. In addition, also the group of genes involved in transcription is more predominant among downregulated genes $(12.7 \%$ versus $6.3 \%$ of the upregulated genes and an intermediate $9 \%$ of the total genome). In concurrence with the trend of genes related to transcription and translation, also genes related to nucleotide biosynthesis are mostly downregulated (42 of 51 genes), yet genes involved in amino acid biosynthesis show a less clear trend (30 down-, 9 upregulated, 54 not differentially expressed). The observed downregulation of major anabolic processes most likely is related to the reduction in doubling time $\left(t_{d}\right.$ of cells on methanol relative to growth on glucose $\left(t_{d}\right.$ methanol $=4.5 \mathrm{~h}, t_{d}$ glucose $=1 \mathrm{~h}$ ) and may reflect the accompanying decrease in DNA replication, RNA transcription and translation.

Stress response genes form only relatively small categories among both the upregulated genes relative to the downregulated genes (2.9\% vs $1.6 \%)$. Based on the Functional Catalogue, only 185 of the nearly 6000 annotated $H$. polymorpha genes are indicated as stress response genes. However, based on several studies by Gasch in Saccharomyces cerevisiae [9-11], many more genes could contribute to the cellular stress response. Hence, most likely also genes classified in other groups play a role in coping with stress accompanying a shift in cultivation conditions. Thus, the contribution of the stress response to the total differential expression in $H$. polymorpha upon transfer to methanol medium is probably larger than the observed $2.9 \%$ upregulation and $1.6 \%$ downregulation. 

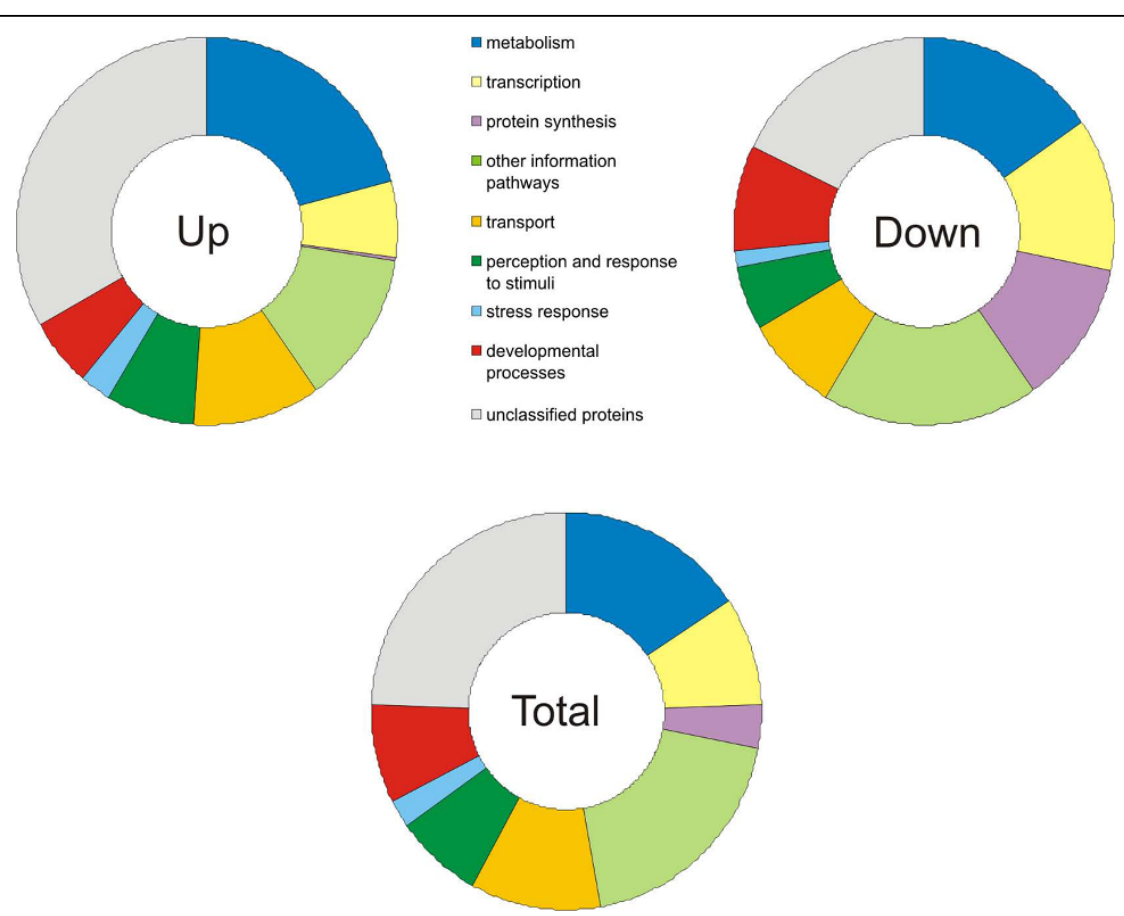

Figure 2 FUNCATS. Representation of functional groups among up- and downregulated genes is shown in a diagram. For comparison, a similar diagram is made for the total number of genes in Hansenula polymorpha (up-, down- and non-regulated genes). Genes are classified in one or multiple groups based on the Functional Catalogue.

A last remarkable group in the Functional Catalogue diagram is the category of unclassified proteins, showing that $33 \%$ of the upregulated genes and $17.9 \%$ of the downregulated genes are thus far not experimentally characterized, relative to $25 \%$ of the genes of the total genome of $H$. polymorpha. This observation suggests that our current knowledge on adaptation to methanol is far from complete.

\section{Metabolic pathways - upregulation of methanol metabolism}

As expected, genes involved in methanol metabolism are highly upregulated. In figure 3 an overview of the methanol metabolism, including the microarray data, is presented [2]. In peroxisomes, methanol is oxidized to formaldehyde and hydrogen peroxide by alcohol oxidase (AOX), which is 17.3 times upregulated at the transcriptional level. The hydrogen

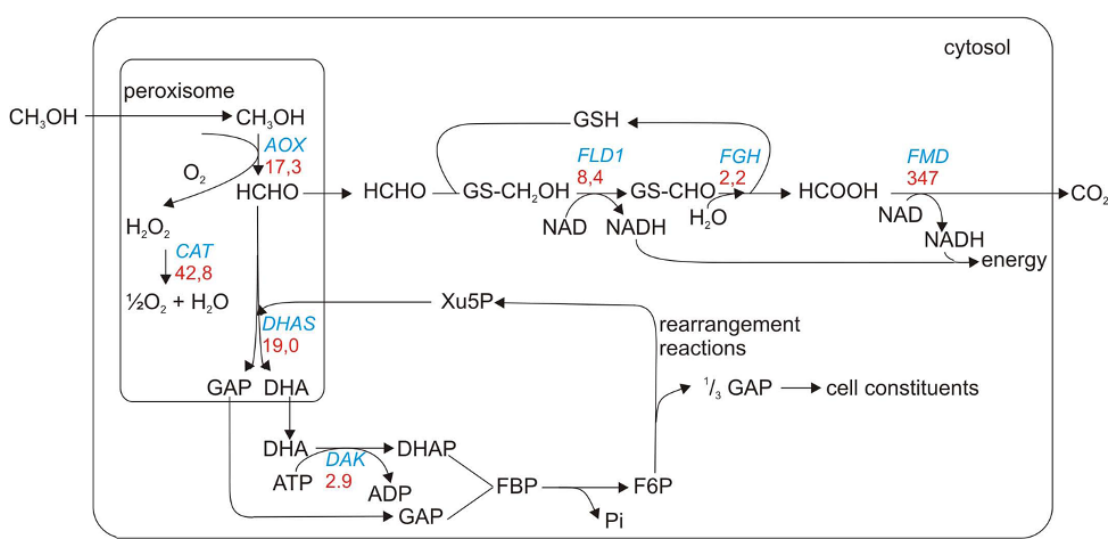

Figure 3 Methanol metabolism. Overview of the methanol metabolism in H. polymorpha. The fold upregulation of the indicated genes is shown in red. GAP: glyceraldehyde-3-phosphate; DHA: dihydroxyacetone; DHAP: dihydroxyacetone phosphate; FBP: fructose bisphosphate; F6P: fructose-6-phosphate; XU5P: xylulose-5-phosphate; GSH: reduced glutathione; GS-CH $2 \mathrm{OH}$ : S-hydroxymethylglutathione; GS-CHO:

S-formylglutathione. AOX: alcohol oxidase; CAT: catalase; DHAS: dihydroxyacetone synthase; DAK: dihydroxyacetone kinase; FLD: formaldehyde dehydrogenase; FGH: S-formyl glutathione hydrolase and FMD: formate dehydrogenase. 
peroxide is detoxified by catalase (CAT) to water and oxygen (42.8-fold upregulated). Formaldehyde can be further metabolized via two different routes: 1) dissimilation via formaldehyde dehydrogenase (FLD1, 8.4-fold up), $\mathrm{S}$-formyl glutathione hydrolase ( $\mathrm{FGH}, 2.2$-fold up) and formate dehydrogenase (FMD, 347-fold up) to $\mathrm{CO}_{2}$, generating $\mathrm{NADH}$ and $\mathrm{CO}_{2}$ or 2) assimilation via the peroxisome-borne enzyme dihydroxyacetone synthase (DHAS, 19,0-fold up) to generate cell constituents. DHAS is part of the xylulose-5-phosphate cycle and catalyzes the formation of two C3-molecules (dihydroxyacetone and glyceraldehyde-3-phosphate) from one $\mathrm{C} 1$ (formaldehyde) and one C5 compound (xylulose-5-phosphate) [2].

Promoter studies in Candida boidinii using phosphatase as a reporter enzyme revealed that upon a shift to methanol medium $F M D$ was induced first, followed by $D H A S$ and even later $A O X$ [12]. The early induction of $F M D$ (347-fold up 2 hours after the shift to methanol medium) relative to $A O X$ and DHAS (17.3-, 19-fold up respectively) suggests that a similar induction pattern may exist in $H$. polymorpha. The differences in induction of the genes 2 hours after the shift to methanol medium does not reflect the ultimate protein levels in the cells, as AOX and DHAS are the major protein bands in extracts prepared from methanol grown $H$. polymorpha cells $[12,13]$.

\section{PEX genes}

$P E X$ genes control the development and function of a specialized class of organelles, the peroxisomes. Most of the PEX genes showed a moderate induction upon the shift to methanol (up to 4-fold; Table 1). This is in line with earlier studies of $S$. cerevisiae cells that were shifted from glucose to the peroxisome-inducing carbon source oleate $[14,15]$. Of the $P E X$ genes involved in import of peroxisomal matrix enzymes (AOX, DHAS and CAT), the highest upregulation was observed for PEX1, PEX4, PEX5, PEX13, PEX14 and PEX26, which all encode key components PTS1 protein import machinery [16]. Highest upregulation was observed for PEX11 (4.7-fold) and PEX32 (4.8-fold). Pex11p, together with Pex25p and Pex11cp, are the three members of the Pex11p protein family in $H$. polymorpha [16]. These proteins are implicated in regulating peroxisome proliferation. Also in bakers' yeast cells shifted from glucose to oleic acid medium PEX11 increased by far the most [14].

H. polymorpha PEX25 was upregulated 2.2-fold, whereas PEX11C, whose function is still unknown, showed a 1.6-fold downregulation [16].

Pex $32 p$ is a member of the Pex 23 protein family, a group of membrane proteins with unknown function [16]. Y. lipolytica pex23 mutants cannot grow on oleate and partially mislocalize peroxisomal proteins to the cytosol, suggesting a role in matrix protein import. In contrast however, S. cerevisiae Pex23p, Pex31p and
Table 1 Expression changes of PEX genes

\begin{tabular}{|c|c|c|}
\hline PEX genes & & Ratio \\
\hline Hp46g103 & PEX 1 & 2.9 \\
\hline Hp24g603 & PEX2 & 1.7 \\
\hline Hp47g896 & PEX3 & 1.5 \\
\hline Hp13g30 & PEX4 & 3.1 \\
\hline Hp28g69 & PEX5 & 3.3 \\
\hline Hp33g316 & PEX6 & 1.6 \\
\hline Hp15g912 & PEX7 & 1.8 \\
\hline Hp27g144 & PEX8 & 1.7 \\
\hline Hp6g229 & PEX10 & 1.6 \\
\hline Hp24g562 & PEX11 & 4.7 \\
\hline Hp5g555 & PEX11C & -1.6 \\
\hline Hp39g539 & PEX12 & 2.6 \\
\hline Hp32g232 & PEX13 & 3 \\
\hline Hp24g522 & PEX14 & 3.5 \\
\hline Hp14g184 & PEX17 & 2.1 \\
\hline Hp9g314 & PEX19 & 1.1 \\
\hline Hp11g43 & PEX20 & 1 \\
\hline $\mathrm{Hp37g108}$ & PEX22 & -1.3 \\
\hline Hp39g248 & PEX23 & 1.2 \\
\hline Hp25g249 & PEX23-like & -1.2 \\
\hline Hp47g626 & PEX24 & 2.9 \\
\hline Hp16g88 & PEX25 & 2.2 \\
\hline Hp15g17 & PEX26 & 3.6 \\
\hline Hp29g7 & PEX29 & -1.2 \\
\hline Hp27g236 & PEX32 & 4.8 \\
\hline
\end{tabular}

All genes shown have a p-value below 0.05 . Negative values indicate downregulation on methanol, positive values indicate upregulation on methanol

Pex32p are not required for protein import but play a role in peroxisome proliferation. Where ScPex23p appears to be a positive regulator of peroxisome size, ScPex31p and ScPex32p have been suggested to negatively regulate this process. The function of $H$. polymorpha Pex32p is not yet known. Based on our current study this protein may be, together with Pex11p, responsible for the initial increase in size of the peroxisomes, as was observed by detailed ultrastructural analysis (figure 4) and in concurrence with earlier findings [5]. The relatively high induction of this peroxin makes it an interesting candidate for further study in $H$. polymorpha.

\section{Metabolic pathways - downregulation of glucose} utilisation

As depicted in Table 2, the majority of the genes involved in glycolysis are downregulated (-1.2 to -5.7). Since the genes are listed in order of appearance in the pathway, it is evident that the strongest downregulation is observed in the steps before fructose 1,6 bisphosphate aldolase. This corresponds with the fact that the 

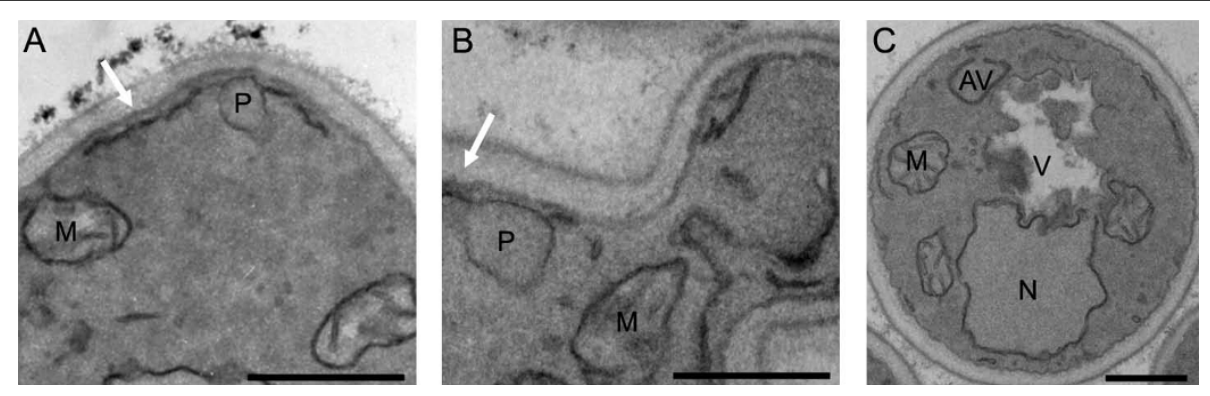

Figure 4 Ultrastructural analysis of the adaptation of cells to methanol. Glucose-grown H. polymorpha WT cells were extensively analysed at different time-points after the shift to methanol by electron microscopy of $\mathrm{KMnO}_{4}$-fixed samples. (A) Control glucose-grown cell and (B) after $2 \mathrm{~h}$ of incubation in the presence of methanol. A clear increase in peroxisome size was observed, cross-sections of representative cells are depicted. Note the association of the organelles with strands of ER (indicated by arrow). (C) 2 hours after the shift a clear increase was observed in large vacuolar autophagic bodies, indicative of induction of autophagy. N - Nucleus, M - Mitochondrion, V - Vacuole, AV - Autophagosome. The bar represents $0.5 \mu \mathrm{m}$.

products of methanol metabolism, dihydroxyacetone and glyceraldehyde 3 phosphate, enter the glycolytic pathway directly after this step, so the subsequent enzymes are still required for progression with the pathway towards the TCA cycle. The observed mild decrease in expression of their encoding genes can be attributed to the reduction in the substrate flow, when switching from glucose to methanol utilisation. However, it should be noted that the enzymes of the final part of glycolysis in majority also function in the direction of gluconeogenesis, by catalyzing the reverse reactions. Finally, the upregulation of the gene encoding fructose 1,6 bisphosphate aldolase which, on methanol, catalyzes the formation of fructose 1,6 bisphosphate from dihydroxyacetone and glyceraldehyde-3-phosphate, reflects an increase of this reaction, which has been shown to be important in the rearrangement reactions to replenish the cell with xylulose-5-phosphate to the downstream reactions in methanol metabolism [2].

\section{Regulatory networks}

Accompanying the changes in expression of many metabolic genes, also changes in the underlying regulatory networks are expected. In addition to global changes, the DNA microarray data reflect the initial adaptation to methanol and thus enable the investigation of more specific changes resulting in activation of methanoldependent genes or in repression of glucose-dependent genes.

Among the upregulated genes, 49 are involved in regulation of transcription. Most of these encode general transcription factors or transcription factors which have not been linked to a specific process. Regulators involved in stress response (6), glucose sensing/derepression (4), and peroxisome-related pathways (4) are overrepresented, as is expected due to the change in carbon source.

The expression of genes coding for peroxisome assembly and function is controlled by a transcriptional regulatory network, which has been studied extensively in $S$. cerevisiae in response to oleate [17-19]. The Oaf1-Pip2 complex plays a prominent role, although the putative $H$. polymorpha homolog of PIP2 is only moderately upregulated during adaptation to methanol. However the homolog of ADR1 (23.7-fold), a second activator of oleate-responsive genes is strongly upregulated in $H$. polymorpha and is also involved in regulation of the response to both oleate and methanol in $P$. pastoris (named MXR1; [20]. Virtually all known targets of

Table 2 Expression changes of glycolysis and gluconeogenesis genes

\begin{tabular}{llc}
\hline Gene & Function & Ratio \\
\hline Glycolysis & & \\
\hline Hp25g374 & Hexokinase & -5.7 \\
\hline Hp24g239 & Glucokinase & -2.3 \\
\hline Hp33g380 & Glucose-6-phosphate isomerase & -1.7 \\
\hline Hp1g417 & Phosphofructokinase alpha subunit & -2.9 \\
\hline Hp39g214 & Phosphofructokinase beta subunit & -2 \\
\hline Hp47g1019 & Fructose 1,6-bisphosphate aldolase & 2.1 \\
\hline Hp16g222 & Triosephosphate isomerase & -1.5 \\
\hline Hp25g306 & Glyceraldehyde-3-phosphate & -1.4 \\
\hline & dehydrogenase & -1.3 \\
\hline Hp26g207 & 3-phosphoglycerate kinase & $1.2^{*}$ \\
\hline Hp37g8 & Phosphoglycerate mutase & -1.7 \\
\hline Hp27g405 & Phosphopyruvate hydratase (enolase) & -1.2 \\
\hline Hp39g227 & Pyruvate kinase & -1.3 \\
\hline Hp6g262 & Pyruvate dehydrogenase, alpha subunit & -1.8 \\
\hline Hp37g184 & Pyruvate dehydrogenase, beta subunit \\
\hline Gluconeogenesis & Pyruvate carboxylase & 1.9 \\
\hline Hp18g102 & Phosphoenolpyruvate carboxykinase & 8.9 \\
\hline Hp5g547 & Fructose 1,6 bisphosphatase & 4.4 \\
\hline Hp46g88 & & \\
\hline
\end{tabular}

* With the exception of the one marked with an asterisk, all genes shown have a $\mathrm{p}$-value below 0.05 . 
Adr1 and its co-regulator Cat8 were indeed also upregulated in $H$. polymorpha, suggesting an important role in regulation of methanol metabolism, while most probably also activation by Snf1 is initiated after the shift, since this global regulator is crucial for growth on non-fermentable carbon sources [21,22]. Mpp1, another transcriptional regulator of methanol metabolism in H. polymorpha, is encoded by the strongest upregulated gene of this study (Hp27g360, 394-fold up), thus suggesting it is a master regulator of methanol-responsive genes [23].

Swi1 and Snf2 also belong to a regulatory complex involved in gene expression of methanol-related genes as well as peroxisomal assembly, however their encoding genes were not induced in the early adaptation phase to methanol [24].

Among the downregulated genes, the decreased transcription of RAP1 (Hp16g154, -3.1) is remarkable. This transcriptional regulator is known to activate transcription of genes encoding ribosomal proteins $[25,26]$ and its downregulation is consistent with the observed massive decrease in transcripts for ribosomal proteins. Interestingly, this gene is also shown to be repressed during the environmental stress response in S. cerevisiae as described by Gasch et al., [9].

\section{Autophagy}

Adaptation of $H$. polymorpha cells to methanol requires a major rearrangement of the cellular architecture. The finding that most $A T G$ genes, which are involved in autophagy and autophagy related processes [27], as well as several proteasomal genes are upregulated (18 up vs 2 down), suggests that cellular reorganisation requires massive degradation of cellular components. Interestingly, the highest upregulation is observed for ATG8 and ATG11 (Table 3). Atg8 has a prominent role in various selective and non-selective macroautophagic processes, whereas Atg11 is specifically involved in selective macroautophagy [28,29]. The function of HpAtg19-like, of which the encoding gene is also upregulated, is not known. However, HpAtg19-like is not involved in selective degradation of peroxisomes (unpublished data). Remarkably only ATG25 is significantly downregulated. Atg25 is involved in selective peroxisome degradation by macropexophagy, but not in microautophagy [29].

Ultrastructural analysis of cells at different time-points after the shift indeed showed strong morphological evidence for increased autophagy, reflected in the massive uptake of cytoplasmic components in the vacuole (figure 4).

Recent findings showed the importance of autophagy during methanol adaptation of $P$. pastoris, not only for cell remodeling, but also to provide amino acids [30]. Consistent with these findings, we also observed that $H$. polymorpha atg mutants showed a slight delay in methanol adaptation (data not shown).
Table 3 Expression changes of ATG genes

\begin{tabular}{|c|c|c|}
\hline \multicolumn{2}{|c|}{ Autophagy-related genes } & \multirow{2}{*}{$\begin{array}{c}\text { Ratio } \\
3.7\end{array}$} \\
\hline Hp24g929 & ATG1 & \\
\hline Hp15g1008 & ATG2 & 2.6 \\
\hline $\mathrm{Hp} 42 \mathrm{~g} 317$ & ATG3 & 3.9 \\
\hline Hp24g999 & ATG4 & 2.3 \\
\hline $\mathrm{Hp} 47 \mathrm{~g} 352$ & ATG5 & 1.8 \\
\hline $\mathrm{Hp} 24 \mathrm{~g} 284$ & ATG6 & 3.7 \\
\hline Hp19g8 & ATG7 & 2.2 \\
\hline $\mathrm{Hp} 48 \mathrm{~g} 37 \mathrm{bm}$ & ATG8 & 6.2 \\
\hline Hp16g127 & ATG9 & 1.6 \\
\hline $\mathrm{Hp} 24 \mathrm{~g} 546 \mathrm{~m}$ & ATG10 & 2.5 \\
\hline $\mathrm{Hp} 25 \mathrm{~g} 507$ & ATG11 & 5.2 \\
\hline Hp33g43 & ATG12 & 2.3 \\
\hline Hp19g348 & ATG13 & 1.6 \\
\hline Hp47g589 & ATG15 & 2.2 \\
\hline $\mathrm{Hp} 24 \mathrm{~g} 680 \mathrm{~m}$ & ATG16 & 1.7 \\
\hline Hp8g289 & ATG17 & $1.1^{*}$ \\
\hline $\mathrm{Hp} 25 \mathrm{~g} 289$ & ATG18 & 1.9 \\
\hline $\mathrm{Hp} 13 \mathrm{~g} 64$ & ATG19-like & 6.6 \\
\hline Hp16g331 & ATG20 & -1.1 \\
\hline $\mathrm{Hp} 44 \mathrm{~g} 480$ & ATG21 & 1.8 \\
\hline Hp18g58 & ATG22 & 1.7 \\
\hline Hp33g356 & ATG24 & 2.8 \\
\hline $\mathrm{Hp} 39 \mathrm{~g} 230$ & ATG25 & -4.3 \\
\hline Hp15g447 & ATG26 & 2.1 \\
\hline Hp39g339 & ATG27 & -1.7 \\
\hline $\mathrm{Hp} 47 \mathrm{~g} 741$ & ATG28 & 1.8 \\
\hline Hp32g359 & ATG30 & 4.1 \\
\hline
\end{tabular}

*With the exception of the one marked with an asterisk, all genes shown have a p-value below 0.05 .

\section{$\beta$-oxidation of fatty acids}

A significant upregulation of genes encoding proteins related to $\beta$-oxidation of fatty acids was observed $[31,32]$. This unexpected cluster is listed in Table 4 . The regulation of these genes could be merely due to derepression as a result of decreasing glucose levels. However we consider this less likely since the observed ratio's and signals are quite substantial. Another explanation could be coregulation of multiple peroxisomal pathways by common regulators. A third option is the increase in cellular fatty acid levels, the substrate for peroxisomal $\beta$-oxidation. This might originate from the observed autophagy, leading to recycling of intracellular membrane lipids.

\section{Mitochondria}

Remarkably, the shift of cells from glucose to methanol is associated with a significant increase in mitochondrial volume fractions (figure 5). Several functional links exist between peroxisomes and mitochondria, both for 
Table 4 Expression changes of genes related to fatty acid $\beta$-oxidation

\begin{tabular}{llc}
\hline Gene & Function & Ratio \\
\hline Hp8g534 & Peroxisomal ABC-transporter sub-unit 1 & 8 \\
\hline Hp33g390 & Peroxisomal ABC-transporter sub-unit 2 & 6.4 \\
\hline Hp44g158 & Adenine nucleotide transporter & 3 \\
\hline Hp33g132 & Fatty-acyl coenzyme A oxidase & 14 \\
\hline Hp8g261 & Multifunctional enzyme & 21.6 \\
\hline Hp24g1381 & 3-ketoacyl-CoA thiolase & 16.3 \\
\hline Hp27g292 & Catalase & 42.8 \\
\hline Hp29g305 & Isocitrate lyase & 30.8 \\
\hline Hp43g61 & Malate synthase & 8.8 \\
\hline Hp36g14 & Isocitrate dehydrogenase & 27.7 \\
\hline Hp47g959 & Carnitine acetyl-CoA transferase & 41.6 \\
\hline Hp39g121 & Carnitine acetyltransferase, YAT1 & 47.1 \\
\hline Hp8g466 & Carnitine acetyltransferase, YAT2 & 36 \\
\hline Hp15g677 & Mitochondrial carnitine/acyl carnitine carrier & 111.3 \\
\hline Allgenes shown have a p-value below 0.05.
\end{tabular}

All genes shown have a p-value below 0.05 .

metabolic pathways and for biogenesis of both organelles $[33,4]$. Of the genes involved in mitochondrial function or assembly 110 are down- and 67 are upregulated. Genes coding for components of the Dnm1dependent fission machinery of both organelles are not differentially expressed [4]. Similar to all downregulated genes, of the mitochondrial downregulated genes almost $50 \%$ is involved in transcription and translation processes. In addition the genes coding for TOM and TIM protein import complexes are also mostly down-regulated. Genes involved in FeS cluster, heme biosynthesis and cytochrome c assembly are overrepresented among upregulated mitochondrial genes (9-fold), in agreement with the prominent role for mitochondria as the sole site of ATP generation during methanol-metabolism [2]. Heme is also the co-factor of peroxisomal catalase which is highly induced. FeS cluster formation is also coupled to the glutathione-based redox regulation system via GRX5 [34].

\section{Reactive oxygen species}

Although mitochondria were long considered as the main source of reactive oxygen species (ROS), also peroxisomes actually defined as organelles that harbour $\mathrm{H}_{2} \mathrm{O}_{2}$-producing enzymes as well as catalase are now recognized as a significant contributor to ROS production $[2,35,36]$. Besides catalase (42.8-fold up), peroxisomes also contain the peroxiredoxin Pmp20, that is 11-fold upregulated, both indicative of an increase in peroxisomal ROS production [37,38].

We also observed an increase of several pivotal genes involved in cytosolic and mitochondrial ROS detoxification; like the superoxide dismutase SOD1 (4.3-fold), the glutathione transferase GTO2 (2.6-fold), the glutathione

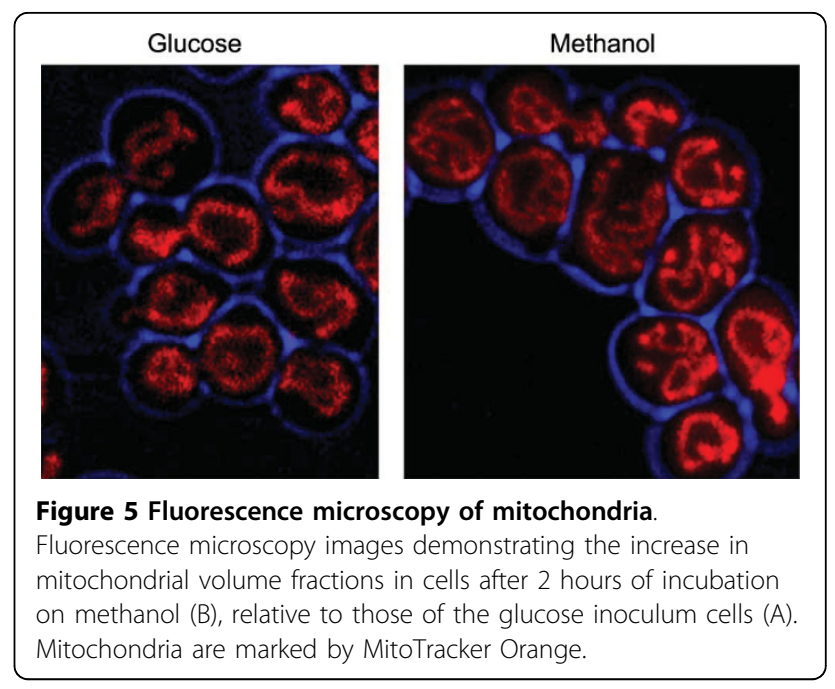

reductase GPX3 (8-fold), the glutaredoxin GRX5 (2.8fold) and the thioredoxin peroxidase TSA1 (47.2-fold). The remaining members of the glutathione- and TRXbased system are not differentially expressed (8 other genes found), except for the thioredoxin reductase TRR1 (10.4-fold). Induction of these key enzymes involved in sustaining the redox balance of the cytosol, suggests that methanol-metabolism also results in enhanced cytosolic ROS levels, which may originate from peroxisomal metabolism.

Comparison with other DNA microarray analyses

Back in 1996, the proof of principle for the use of DNA microarray technology to investigate transcriptional changes was shown for S. cerevisiae [39]. Since then, DNA microarray analysis has become a regular, wellestablished tool, facilitated by the now commercial available array slides. For many other yeast species however, thusfar the usage of DNA microarray analysis is still rather limited. Only recently, species-specific DNA microarray studies have been presented for e.g. Debaryomyces hansenii [40] and P. pastoris [41]. For H. polymorpha, Oh et al., [42] presented a partial, DNA microarray study, followed by a full DNA microarray analysis describing the transcriptional response of $H$. polymorpha to exposure to cadmium [43]. However, this study was not related to the metabolic and architectural alterations associated with a change in cellular metabolism.

Smith et al. [15,18] published two studies in which bakers' yeast was shifted to oleate. The first study focused mainly on transcriptional changes of genes encoding peroxisomal proteins and peroxins, the second one on the regulatory network coordinating the response to oleate.

The current study is the first in which arrays were used to study the shift from glucose to methanol in the yeast $H$. polymorpha. Sauer et al. [44] was the first to 
publish transcriptional profiling of the methylotrophic yeast $P$. pastoris, also upon a shift to methanol. However, in that study, yeast cells were transferred from glycerol to methanol and heterologous hybridisation onto S. cerevisiae DNA-microarrays was performed. Still, the same trend in regulated functional groups was observed. Also, similar effects were reported on carbohydrate metabolism and the regulation of ribosomal genes. Although some of the data obtained for H. polymorpha are comparable with those obtained for $P$. pastoris, a significant drawback of the latter study is the absence of $P$. pastoris specific or methylotrophic yeast specific genes on the DNA-microarray slides. There is indeed a substantial amount of methanol-responsive $H$. polymorpha genes found in our study which were not observed in $P$. pastoris using $S$. cerevisiae microarrays $( \pm 450$ upregulated, \pm 350 downregulated) [Additional file 2: Supplemental Table S2].

\section{Conclusions}

The current DNA microarray study revealed the expected repression of genes involved in glucose metabolism concomitant with the induction of the genes of methanol metabolism in response of a shift of $H$. polymorpha cells from glucose to methanol. Also genes involved in peroxisome biogenesis (PEX genes) are upregulated, with $P E X 32$ being the most strongly upregulated $P E X$ gene. A surprising finding was the upregulation of autophagyand of beta-oxidation-genes. The first can likely be explained by the need for cellular reorganisations and is confirmed by electron microscopy studies showing active uptake of cytoplasmic components in the vacuoles of the cells. The induction of beta-oxidation is thought to be a consequence of the cellular reorganisations and thereby the high turnover of lipids, serving as substrates for betaoxidation. A final interesting but not yet uncovered group consists of the $33 \%$ of the upregulated genes that have no known function. These genes reflect a large potential of $H$. polymorpha or methylotrophic yeast specific genes with a specialized role in adaptations to growth on methanol as the sole source of carbon and can form interesting targets for future research.

\section{Methods}

\section{H. polymorpha microarray probe design}

$H$. polymorpha open reading frame sequences were collected from the $H$. polymorpha genome database (Rhein Biotech, unpublished; [7] and NCBI [45-47]. For the genes on contigs $1-48$, the annotation was based on the information from RheinBiotech and Ramezani-Rad et al. [7]. The additional NCBI sequences were listed as Hp50 and $\mathrm{Hp} 51$ numbers. The annotation of these genes was as described on NCBI (Hp50s) or by manual blast search (Hp51s). All Hp sequences were applied for design of oligonucleotide probes using OLIGOARRAY v2.1 [48] with the following oligonucleotide primer design parameters: a length of 58-60 nucleotides, a melting temperature $(\mathrm{Tm})$ of at least $80^{\circ} \mathrm{C}$, a G/C-content of $34-52 \%$ and a maximum $\mathrm{Tm}$ of secondary structures and cross-hybridisations of $68^{\circ} \mathrm{C}$. Oligo's were designed within the 3'regions of the ORFs (setting: maximum distance between the $5^{\prime}$ end of the oligo and the 3' end of the input sequence: 600-nt) to minimise including intronsequences, since these were not discarded in the input ORF sequences. Paralogous sequences were removed during the final analysis of the design using blastN. Of the 6,248 oligo-probes, 6,002 are from the annotated genes of $H$. polymorpha and 23 probes are from heterologous genes of specific research interest (data not shown). The remaining 223 probes include positive and negative controls. Subsequently, the oligo-set was printed twice in each of the 8 arrays per slide (8-plex format) using Agilent's SurePrint technology (in situ synthesis; via eArray 4.0-website; Agilent Technologies Netherlands B.V., Amstelveen, the Netherlands).

\section{Organisms \& Growth}

H. polymorpha strain NCYC $495 \mathrm{leu}^{-}$was grown in batch cultures on mineral medium containing either $0.5 \%$ glucose or $0.5 \%$ methanol as carbon source and $0.25 \%$ ammonium sulphate as nitrogen source $[49,50]$. For transcriptome analysis, cells were extensively precultivated in batch cultures on glucose at $37^{\circ} \mathrm{C}$ prior to a shift to fresh media containing methanol as sole carbon source. Four independent glucose-grown cultures were used to inoculate fresh medium containing methanol as well as for taking samples for RNA isolation. The methanol cultures were grown for two hours, followed by RNA isolation.

\section{RNA isolation}

Samples were harvested by freezing them directly in liquid nitrogen, followed by thawing on ice, centrifugation $\left(5.000 \mathrm{G}, 4 \mathrm{~min}, 0^{\circ} \mathrm{C}\right.$ ) and taken up in AE-buffer (50 mM sodium-acetate $10 \mathrm{mM}$ EDTA pH 5.0). 1 volume acid-phenol chloroform isoamylalcohol (125:24:1 $\mathrm{pH} 4.5$, Ambion, Austin USA) and 0.5\% SDS was added, and incubated at $65^{\circ} \mathrm{C}$ for $5 \mathrm{~min}$ followed by $15 \mathrm{~min}$ at $-80^{\circ} \mathrm{C}$. After centrifugation ( $\left.15 \mathrm{~min} 13.000 \mathrm{G}\right)$, the upper phase was mixed with 0.5 volume acid phenol/chloroform, centrifuged $(4 \mathrm{~min} 13.000 \mathrm{G}$ ) and mixed with 0.5 volume chloroform. The upper phase was used for RNA isolation using column purification according to the manufacturers' instructions (Nucleospin RNA II, Macherey-Nagel, Düren Germany). The Agilent Bioanalyzer 2100 with RNA 600 Nano chips was used to analyze the quality and integrity of the RNA samples.

\section{Biochemical methods}

Transcript levels of methanol-related genes $(A O X$ and $D H A S)$, using those of actin as control, were determined 
by semi-quantitative RT-PCR, using actin as loading control (Ready-to-go RT-PCR beads, GE Healthcare, Little Chalfont UK).

Primers:

AOX-forw: CGTGAGAACAGTGCCAATGAAG

AOX-rev: TCACCGATGGTCAATGCAGTAG

DHAS-forw: GCAGGACGTGTACGACTTCTTC

DHAS-rev: GTAGGACGCCGTAGCGTATCTC

Act1-forw: GGTCATTGATAACGGATCCGG

Act1-rev: CACTTGTGGTGGACAATGGATGG

Cell lysates were essentially obtained as described [51], for subsequent AOX activity measurements as described [52].

DNA microarray analysis - labeling, hybridisation, washing and scanning

Using the Low RNA input linear Amplification kit from Agilent, cDNA was generated based on $500 \mathrm{ng}$ of each isolated mRNA sample. Next, cRNA was made using Cy3-CTP or Cy5-CTP incorporation for labeling purposes. For each original mRNA, a portion of $\mathrm{Cy} 3$ and Cy5 labeled cRNA was generated. The concentration and incorporation of the cRNA and the dyes are measured using Nanodrop. For each biological replicate, 300 ng Cy3 labeled glucose culture originating cRNA was used for hybridisation against $300 \mathrm{ng}$ Cy5 labeled methanol culture originating cRNA and vice versa for the dye-swap, resulting in 8 hybridisations in total. Hybridisation, washing and scanning were performed according to the Agilent 'Two-color Microarray-based gene expression analysis protocol' (version 5.5, February 2007) by ServiceXS (Leiden, The Netherlands).

Data analysis - hybridisation ratio's, A-values and p-values

To extract the data from the scanned DNA-microarray slides, the feature extraction software version 9.5, Protocol GE2-v5_95_Feb07 from Agilent was used. For the background subtraction the option 'No background subtraction and spatial detrend' was used. For each spot, the ratio between the green and red processed signals was calculated, reflecting the ratio of gene expression on methanol overexpression on glucose. Next, the average ratio per gene was calculated based on the data of 16 spots (8 hybridisations, 2 spots per hybridisation). For reasons of clarity, genes with a ratio of $<1$ were expressed as -( 1 divided by the ratio), thus reflecting the fold downregulation (e.g. -2 instead of 0.5). As a cut-off for differential gene expression, a threshold of (more than) 2-fold up- or downregulation was used, so $>2$ or $<-2$. To assess the significance of the data, $\mathrm{p}$-values were computed using the paired-data program at the CyberT interface $[53,54]$. Genes were considered to be significantly regulated if they had a p-value below 0.05 . In addition, average A-values for each gene were calculated as an indication for the intensity of the signals using
$\mathrm{A}=0.5^{*}(\log 2 \mathrm{Cy} 3+\log 2 \mathrm{Cy} 5)$. An A-value of 6 was used as a lower limit for trustworthy signal intensity.

All data has been deposited to the NCBI Gene expression omnibus and is accessible under accession number GSE19036.

\section{Classification according to the Functional Catalogue}

To show the main represented functions among up- and downregulated genes, $H$. polymorpha genes were ordered according to their Functional Catalogue (FunCat) as assigned by RheinBiotech. In the diagrams, the main groups of the hierarchical structure are shown as well as the subgroups 'transcription' and 'protein synthesis' [8]. The group 'subcellular localisation' was omitted, while 'control of cellular organisation', which is not in the FunCat structure, was included under 'developmental processes'. Genes can be present in more than one group.

\section{Analysis of metabolic routes using Biocyc}

Changes in expression of metabolic pathway genes were investigated using the omics viewer at the Ecocyc website [55]. Since information on $H$. polymorpha is not included in this database, the genome of Saccharomyces cerevisiae S288C was used as a reference.

\section{Microscopy}

Ultrathin sections of $\mathrm{KMnO}_{4}$-fixed cells were used for ultrastructural analysis as described [56]. Analysis of mitochondria was performed using confocal microscopy (Zeiss LSM510). Mitochondria were visualized using MitoTracker Orange (CMTMRos, Invitrogen) and visualization with excitation by a $543 \mathrm{~nm}$ Neon-laser (Lasos) and detection using a 565-615 band-pass emission filter.

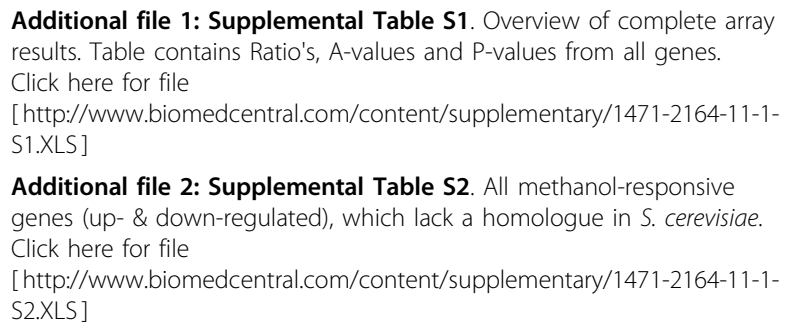

Additional file 2: Supplemental Table S2. All methanol-responsive genes (up- \& down-regulated), which lack a homologue in S. cerevisiae. Click here for file

[ http://www.biomedcentral.com/content/supplementary/1471-2164-11-1S2.XLS ]

\section{Acknowledgements}

TVZ is supported by a grant from the Netherlands Organisation for Scientific Research NWO (Earth and Life Sciences). This project was carried out within the research programme of the Kluyver Centre for Genomics of Industrial Fermentation, which is part of the Netherlands Genomics Initiative/ Netherlands Organisation for Scientific Research.

We thank Rhein Biotech GmbH, Düsseldorf, Germany for access to the H. polymorpha genome database.

\section{Author details}

${ }^{1}$ Molecular Cell Biology, University of Groningen, P.O. Box 14, 9750 AA Haren, the Netherlands. ${ }^{2}$ Molecular Genetics, University of Groningen, P.O. Box 14, 9750 AA Haren, the Netherlands. ${ }^{3}$ Kluyver Centre for Genomics of Industrial Fermentation, P.O. Box 5057, 2600 GA Delft, the Netherlands. 


\section{Authors' contributions}

TVZ was involved in the annotation of the sequences used for the DNA microarray and performed the DNA microarray experiments. Furthermore, he has been active in data analysis and performed the additional experiments. RJSB performed the sequence annotation and designed and performed the DNA microarrays. He also has been involved in the data analysis. KAS has performed the DNA microarray analysis. AdJ has been involved in design of the DNA microarray slides. OPK supervised the array analysis. MV was involved in microscopy analysis and writing of the paper. IJvdK was general supervisor and involved in writing of the paper. All authors have read and approved the final manuscript.

Received: 15 September 2009

Accepted: 4 January 2010 Published: 4 January 2010

\section{References}

1. Gellissen G, Kunze G, Gaillardin C, Cregg JM, Berardi E, Veenhuis M, Klei van der IJ: New yeast expression platforms based on methylotrophic Hansenula polymorpha and Pichia pastoris and on dimorphic Arxula Adeninivorans and Yarrowia lipolytica - A comparison. FEMS Yeast Res 2005, 5:1079-1096.

2. Klei Van der IJ, Yurimoto H, Sakai Y, Veenhuis M: The significance of peroxisomes in methanol metabolism in methylotrophic yeast. Biochim Biophys Acta 2006, 1763:1453-1462.

3. Ischuk OP, Voronovsky AY, Abbas CA, Sibirny AA: Construction of Hansenula polymorpha strains with improved thermotolerance. Biotechnol Bioeng 2009, 104:911-919.

4. Nagotu S, Krikken AM, Otzen M, Kiel JAKW, Veenhuis M, Klei van der IJ: Peroxisome fission in Hansenula polymorpha requires Mdv1 and Fis1, two proteins also involved in mitochondrial fission. Traffic 2008, 9:1471-1484

5. Veenhuis M, Keizer I, Harder W: Characterization of peroxisomes in glucosegrown Hansenula polymorpha and their development after the transfer of cells into methanol-containing media. Arch Microbiol 1979, 120:167-175.

6. Eggeling L, Sahm H: Derepression and Partial Insensitivity to Carbon Catabolite Regression of the Methanol Dissimilating Enzymes in Hansenula polymorpha. Europ J Appl Microbiol Biotechnol 1978, 5:197-202.

7. Ramezani-Rad M, Hollenberg CP, Lauber J, Wedler H, Griess E, Wagner C, Albermann K, Hani J, Piontek M, Dahlems U, Gellissen G: The Hansenula polymorpha (strain CBS4732) genome sequencing analysis. FEMS Yeast Res 2003, 4:207-215.

8. Ruepp A, Zollner A, Maier D, Albermann K, Hani J, Mokrejs M, Tetko I, Güldener U, Mannhaupt G, Münsterkötter M, Mewes HW: The FunCat, a functional annotation scheme for systematic classification of proteins from whole genomes. Nucl Acid Res 2004, 32:5539-5545.

9. Gasch AP, Spellman PT, Kao CM, Carmel-Harel O, Eisen MB, Storz G, Botstein $D$, Brown PO: Genomic expression programs in the response of yeast cell to environmental changes. Mol Biol Cell 2000, 11:4241-4257.

10. Gasch AP, Werner-Washburne M: The genomics of yeast responses to environmental stress and starvation. Funct Integr Genomics 2002, 2:181-192.

11. Gasch AP: Comparative genomics of the environmental stress response in ascomycete fungi. Yeast 2007, 24:961-976.

12. Yurimoto H, Komeda T, Lim CR, Nakagawa T, Kondo K, Kato N, Sakai Y: Regulation and evaluation of five methanol-inducible promoters in the methylotrophic yeast Candida boidinii. Biochim Biophys Acta 2000, 1493: 56-63.

13. Roa M, Blobel G: Biosynthesis of peroxisomal enzymes in the methylotrophic yeast Hansenula polymorpha. Proc Natl Acad Sci USA 1983, 80:6872-6876

14. Kal JA, van Zonneveld AJ, Benes V, Berg van den M, Koerkamp MG, Albermann K, Strack N, Ruijter JM, Richter A, Dujon B, Ansorge W, Tabak HF: Dynamics of gene expression revealed by comparison of serial analysis of gene expression transcript profiles from yeast grown on two different carbon sources. Mol Biol Cell 1999, 10:1859-1872.

15. Smith JJ, Marelli M, Christmas RH, Vizeacoumar FJ, Dilworth DJ, Ideker T, Galitski T, Dimitrov K, Rachubinsky RA, Aitchison JD: Transcriptome profiling to identify genes involved in peroxisome assembly and function. J Cell Biol 2002, 158:259-271.
16. Kiel JAKW, Veenhuis $M$, Klei van der IJ: PEX genes in fungal genomes: common rare or redundant. Traffic 2006, 7:1291-1303.

17. Gurvitz A, Rottensteiner $\mathrm{H}$ : The biochemistry of oleate induction: transcriptional upregulation and peroxisome proliferation. Biochim Biophys Acta 2006, 1763:1392-1402.

18. Smith JJ, Ramsey SA, Marelli M, Marzolf B, Hwang D, Saleem RA, Rachubinski RA, Aitchison JD: Transcriptional responses to fatty acid are coordinated by combinatorial control. Mol Syst Biol 2007, 3:115.

19. Karpichev IV, Durand-Heredia JM, Luo Y, Small GM: Binding characteristics and regulatory mechanisms of the transcription factors controlling oleate-responsive genes in Saccharomyces cerevisiae. J Biol Chem 2008, 283:10264-10275.

20. Lin-Cereghino GP, Godfrey L, de la Cruz BJ, Johnson S, Khuongsathiene S, Tolstorukov I, Yan M, Lin-Cereghino J, Veenhuis M, Subramani S, Cregg JM: Mxr1p, a key regulator of the methanol utilization pathway and peroxisomal genes in Pichia pastoris. Mol Cell Biol 2007, 26:883-897.

21. Young ET, Dombek KM, Tachibana C, Ideker T: Multiple pathways are coregulated by the protein kinase Snf1 and the transcription factors Adr1 and Cat8. J Biol Chem 2003, 278:26146-26158.

22. Tachibana C, Yoo JY, Tagne J-B, Kacherovsky N, Lee TI, Young ET: Combined global localization analysis and transcriptome data identify genes that are directly coregulated by Adr1 and Cat8. Mol Cell Biol 2005, 25:2138-2146.

23. Leão-Helder NA, Krikken AM, Klei van der IJ, Kiel JAKW, Veenhuis M: Transcriptional downregulation of peroxisome numbers affects selective peroxisome degradation in Hansenula polymorpha. J Biol Chem 2003, 278:40749-40756.

24. Ozimek P, Lahtchev K, Kiel JAKW, Veenhuis M, Klei van der IJ: Hansenula polymorpha Swi1 and Snf2 are essential for methanol utilisation. FEMS Yeast Res 2004, 4:673-682.

25. Rotenberg MO, Woolford JL: Tripartite upstream promoter element essential for expression of Saccharomyces cerevisiae ribosomal protein genes. Mol Cell Biol 1986, 6:674-687

26. Woudt LP, Smit AB, Mager WH, Planta RJ: Conserved sequence elements upstream of the gene encoding yeast ribosomal protein $\mathrm{L} 25$ are involved in transcription activation. EMBO J 1986, 5:1037-1040.

27. Klionsky DJ, Cregg JM, Dunn WAJr, Emr SD, Sakai Y, Sandoval IV, Sibirny A, Subramani S, Thumm M, Veenhuis M, Ohsumi Y: A unified nomenclature for yeast autophagy-related genes. Dev Cell 2003, 5:539-45.

28. Shintani T, Huang W-P, Stromhaug PE, Klionsky DJ: Mechanism of cargo selection in the cytoplasm to vacuole targeting pathway. Dev Cell 2002, 3:825-837

29. Monastyrska I, Kiel JAKW, Krikken AM, Komduur JA, Veenhuis M, Klei van der IJ: The Hansenula polymorpha ATG25 gene encodes a novel coiled-coil protein that is required for macropexophagy. Autophagy 2005, 1: 92-100.

30. Yamashita S-I, Yurimoto H, Murakami D, Yoshikawa M, Oku M, Sakai Y: Lagphase autophagy in the methylotrophic yeast Pichia pastoris. Genes Cells 2009, 14:861-870

31. van Roermund CWT, Waterham HR, Wanders RJH: Fatty acid metabolism in Saccharomyces cerevisiae. Cell Mol Life Sci 2003, 60:1838-1851.

32. Zwart KD, Veenhuis M, Plat G, Harder W: Characterization of glyoxysomes in yeasts and their transformation into peroxisomes in response to changes in environmental conditions. Arch Microbiol 1983, 136:28-38.

33. Wanders RJA, Waterham HR: Biochemistry of mammalian peroxisomes revisited. Annu Rev Biochem 2006, 75:295-332.

34. Rodriguez-Manzaneque MT, Tamarit J, Belli G, Ros J, Herrero E: Grx5 is a mitochondrial glutaredoxin required for the activity of iron/sulfur enzymes. Mol Biol Cell 2002, 13:1109-1121.

35. Herrero E, Ros J, Belli G, Cabiscol G: Redox control and oxidative stress in yeast cells. Biochim Biophys Acta 2007, 1780:1217-1235.

36. Bener Aksam E, de Vries B, Klei van der IJ, Kiel JAKW: Preserving organelle vitality: peroxisomal quality control mechanisms in yeast. FEMS Yeast Res 2009, 45:1115-1124.

37. Goodman JM, Maher J, Silver PA, Pacifico A, Sanders D: The membrane proteins of the methanol-induced peroxisome of Candida boidinii. Initial characterization and generation of monoclonal antibodies. J Biol Chem 1986, 261:3464-3468.

38. Bener Aksam E, Jungwirth $H$, Kohlwein SD, Ring J, Madeo F, Veenhuis M, Klei van der IJ: Absence of the peroxiredoxin Pmp20 causes peroxisomal protein leakage and necrotic cell death. Free Radic Biol Med 2008, 45:1115-1124. 
39. Shalon D, Smith SJ, Brown PO: A DNA microarray system for analyzing complex DNA samples using two-color fluorescent probe hybridization. Genome Res 1996, 6:639-645.

40. Gonzalez NA, VázquezA A, Ortiz Zuazaga HG, Sen A, Olvera HL, Peña de Ortiz S, Govind NS: Genome-wide expression profiling of the osmoadaptation response of Debaryomyces hansenii. Yeast 2009, 26:111-124.

41. Graf A, Gasser B, Dragosits M, Sauer M, Leparc GG, Tüchler T, Kreil DP, Mattanovich D: Novel insights into the unfolded protein response using Pichia pastoris specific DNA microarrays. BMC Genomics 2008, 9:390.

42. Oh KS, Kwon O, Oh YW, Sohn MJ, Jung S, Kim YK, Kim MG, Rhee SK, Gellissen G, Kang HA: Fabrication of a partial genome microarray of the methylotrophic yeast Hansenula polymorpha: optimization and evaluation of transcript profiling. J Microbiol 2004, 14:1239-1248.

43. Park JN, Sohn MJ, Oh DB, Kwon O, Rhee SK, Hur CG, Lee SY, Gelissen G, Kang HA: Identification of the Cadmium-inducible Hansenula polymorpha SEO1 gene promoter by transcriptome analysis and its application to whole-cell heavy-metal detection systems. Appl Env Microbiol 2007, 73:5990-6000.

44. Sauer M, Branduardi P, Gasser B, Valli M, Maurer M, Porro D, Mattanovich D: Differential gene expression in recombinant Pichia pastoris analysed by heterologous DNA microarray hybridisation. Microb Cell Fact 2004, 3:17.

45. Blandin G, Llorente B, Malpertuy A, Wincker P, Artiguenave F, Dujon B: Genomic exploration of the hemiascomycetous yeasts: 13 Pichia angusta. FEBS Lett 2000, 487:76-81.

46. Garcia-Lugo P, Gonzalez C, Perdomo G, Brito N, Avila J, de la Rosa JM, Siverio JM: Cloning, sequencing and expression of H.a. YNR1 and H.a. YNI1, encoding nitrate and nitrite reductases in the yeast Hansenula anomala. Yeast 2000, 16:1099-1105.

47. Avila J, Gonzalez C, Brito N, Siverio JM: Clustering of the YNA1 gene encoding a $\mathrm{Zn}(\mathrm{II}) 2 \mathrm{Cys} 6$ transcriptional factor in the yeast Hansenula polymorpha with the nitrate assimilation gene YNT1, YNI1 and YNR1, and its involvement in their transcriptional activation. Biochem J 1998, 335:647-652.

48. Rouillard JM, Zuker M, Gulari E: OligoArray 2.0: design of oligonucleotide probes for DNA microarrays using a thermodynamic approach. Nucl Acid Res 2003, 31:3057-3062

49. Gleeson MAG, Sudbery PE: Genetic analysis in the methylotrophic yeast Hansenula polymorpha. Yeast 1988, 4:293-303.

50. Van Dijken JP, Otto R, Harder W: Growth of Hansenula polymorpha in a methanol-limited chemostat. Physiological responses due to the involvement of methanol oxidase as a key enzyme in methanol metabolism. Arch Microbiol 1976, 111:137-144.

51. Waterham HR, Keizer-Gunnink I, Goodman JM, Harder W, Veenhuis M: Development of multipurpose peroxisomes in Candida Boidinii grown in oleic acid-methanol limited continuous cultures. J Bacterio/ 1992, 174 4057-4063.

52. Verduyn C, van Dijken JP, Scheffers WA: Colorimetric alcohol assays with alcohol oxidase. J Microbiol Methods 1984, 2:15-25.

53. Cyber-T. http://cybert.microarray.ics.uci.edu.

54. Baldi P, Long AD: A Bayesian Framework for the analysis of microarray expression data: regularized t-Test and statistical interferences of gene changes. Bioinformatics 2001, 17:509-519.

55. Ecocyc database. http://ecocyc.org/.

56. Van Zutphen T, Klei van der IJ, Kiel JAKW: Pexophagy in Hansenula polymorpha. Methods Enzymol 2008, 451:197-215.

doi:10.1186/1471-2164-11-1

Cite this article as: van Zutphen et al:: Adaptation of Hansenula polymorpha to methanol: a transcriptome analysis. BMC Genomics 2010 11:1.

\section{Submit your next manuscript to BioMed Central and take full advantage of:}

- Convenient online submission

- Thorough peer review

- No space constraints or color figure charges

- Immediate publication on acceptance

- Inclusion in PubMed, CAS, Scopus and Google Scholar

- Research which is freely available for redistribution 